\section{Reframing the Value of Treatments for Relapsed/Refractory Multiple Myeloma}

In the article "Cost-effectiveness of Drugs to Treat Relapsed/ Refractory Multiple Myeloma in the United States," published in the JMCP January 2018 issue, Carlson et al. seek to identify the most cost-effective therapy for relapsed/refractory multiple myeloma (R/RMM). ${ }^{1}$ Instead, for MM, a chronic, progressive, and heterogenous disease, the decision problem is to identify the regimen sequences that maximize benefit for patients longitudinally over their disease course. Optimal treatment varies by patient at each relapse, since stakeholders need to consider prognostic factors, previous therapies, and associated response (duration/depth); residual adverse events; and, of course, individual patient preferences. In this letter, we discuss limitations of the Carlson et al. analysis that significantly affect the results and conclusions. Inaccurate statements that warrant correction are also identified.

A major limitation stems from the network meta-analysis (NMA), which yields estimates of relative efficacy that are not reliable and valid and lack face validity: lenalidomide (LEN) plus dexamethasone (DEX) is estimated to be less effective than bortezomib (BOR) + DEX, even though all listed trials (Appendix A) show that LEN + DEX achieved double the progression-free survival (PFS) than $\mathrm{BOR}+\mathrm{DEX}^{1}$; panobinostat (PAN) + BOR+DEX is estimated to be more effective than LEN + DEX (PFS hazard ratio of 0.54), even though the appraisal committee for the National Institute of Clinical Excellence (NICE) concluded that there is no difference in effectiveness between $\mathrm{PAN}+\mathrm{BOR}+\mathrm{DEX}$ and $\mathrm{LEN}+\mathrm{DEX}$ in third-line treatment. ${ }^{2}$

Trials included in the NMA are not comparable due to differences in clinical trial designs, populations, and end-points. In CASTOR, ${ }^{3}$ BOR + DEX duration was capped at 6 months and in ENDEAVOR was used to treat-to-progression (TTP), ${ }^{4}$ and a majority of patients $(>50 \%)$ received BOR + DEX beyond 6 months in ENDEAVOR. In its appraisal, the NICE committee concluded that capping treatment duration instead of TTP would reduce BOR+DEX efficacy. ${ }^{5}$

Carfilzomib (CFZ) + DEX, an NCCN preferred category 1 regimen for previously treated MM with proven PFS and overall survival (OS) superiority over BOR+DEX, was not even considered in the Carlson et al. analysis. The cost-effectiveness of CFZ + DEX versus BOR + DEX has been unequivocally established. ${ }^{6}$ For CFZ + LEN + DEX, Carlson et al. appear to overestimate treatment costs by possibly not capping CFZ dosing at 18 cycles per the ASPIRE trial and label, not considering discontinuation of treatments due to reasons other than progression, and by overestimating wastage. Accounting for these corrections would confirm that CFZ + LEN + DEX is cost-effective versus LEN + DEX. ${ }^{7}$

The Jakubowiak et al. (2017) study, which concluded that $\mathrm{CFZ}+\mathrm{LEN}+\mathrm{DEX}$ is cost-effective versus LEN + DEX, was cited inaccurately in several instances. Carlson et al. misreported an odds ratio as a hazards ratio. In Jakubowiak et al., PFS curves for $\mathrm{CFZ}+\mathrm{LEN}+\mathrm{DEX}$ generated by the model aligned very well with the PFS curves observed in the ASPIRE trial (Figure 2), ${ }^{4}$ yet Carlson et al. stated that there is a mismatch between "modeled" and "ASPIRE trial-observed" results probably because they compare a "mean" estimate from the model to the "median" from the ASPIRE trial. Jakubowiak et al. report postprogression survival and costs to be greater for CFZ + LEN + DEX (Table 8), ${ }^{4}$ yet Carlson et al. inaccurately state the opposite.

We strongly recommend that Carlson et al. undertake a reanalysis to address cited limitations and withdraw inaccurate statements. By publishing this letter, JMCP will serve patients well in its mission to present balanced information that contributes to improving the quality of care for patients.

\section{Sikander Ailawadhi, MD}

Division of Hematology/Oncology, Department of Medicine, Mayo Clinic

Jacksonville, Florida

Sumeet Panjabi, PhD

Amgen

South San Francisco, California

Marco Campioni, PhD

Amgen

Zug, Switzerland

Istvan Majer, PhD

Amgen

Zug, Switzerland

Andrzej Jakubowiak, MD, PhD

Myeloma Program

University of Chicago

Chicago, Illinois

\section{DISCLOSURES}

Ailawadhi reports research support from Pharmacyclics and consulting relationships with Takeda, Amgen, and Celgene. Jakubowiak reports consulting and advisory board relationships with AbbVie, Amgen, BMS, Celgene, Karyopharm, SkylineDX, and Takeda. Panjabi, Campioni, and Majer are employees of and stockholders in Amgen.

\section{REFERENCES}

1. Carlson JJ, Guzauskas GF, Chapman RH, et al. Cost-effectiveness of drugs to treat relapsed/refractory multiple myeloma in the United States. J Manag Care Spec Pharm. 2018;24(1):29-38. Available at: https://www.jmcp.org/ doi/10.18553/jmcp.2018.24.1.29

2. National Institute for Health and Care Excellence. Panobinostat for treating multiple myeloma for a least 2 previous treatments. Technology Appraisal Guidance 380. January 27, 2016. Available at: https://www.nice.org.uk/guidance/ta380. Accessed June 6, 2018.

3. Palumbo A, Chanan-Khan A, Weisel K, et al. Daratumumab, bortezomib, and dexamethasone for multiple myeloma. N Engl J Med. 2016;375(8):754-66 
4. Dimopoulos MA, Goldschmidt H, Niesvizky R, et al. Carfilzomib or bortezomib in relapsed or refractory multiple myeloma (ENDEAVOR): an interim overall survival analysis of an open-label, randomised, phase 3 trial. Lancet Oncol. 2017;18(10):1327-37.

5. National Institute for Health for Care Excellence. Carfilzomib for previously treated multiple myeloma. June 2017. Available at: https://www.nice. org.uk/guidance/ta457/documents/final-appraisal-determination-document. Accessed June 6, 2018.

6. Jakubowiak AJ, Houisse I, Májer I, et al. Cost-effectiveness of carfilzomib plus dexamethasone compared with bortezomib plus dexamethasone for patients with relapsed or refractory multiple myeloma in the United States. Expert Rev Hematol. 2017;10(12):1107-19.

7. Jakubowiak AJ, Campioni M, Benedict Á, et al. Cost-effectiveness of adding carfilzomib to lenalidomide and dexamethasone in relapsed multiple myeloma from a U.S. perspective. J Med Econ. 2016;19(11):1061-74.

\section{The Authors Respond}

We appreciate the opportunity to respond to the letter by Ailawadhi et al. regarding our article "Cost-effectiveness of Drugs to Treat Relapsed/Refractory Multiple Myeloma in the United States" and to provide clarifications where necessary to the points raised in their letter.

We do acknowledge 4 minor errors in our publication and have submitted appropriate corrections to JMCP. We will comment on 2 of those errors here, since the third and fourth were merely typos. The first relates to the progression-free survival (PFS) hazard ratio estimate for the panobinostat/ bortezomib/dexamethasone combination (PAN + BOR + DEX) versus lenalidomide (LEN) + DEX in the third-line treatment setting. Our study reported this estimate to be 0.54 (credible range $=0.50-0.83$ ) whereas the correct numbers should be 0.59 (credible range $=0.31-1.10$ ). ${ }^{1}$ This change also results in a slight revision to the comparative $\mathrm{PAN}+\mathrm{BOR}+\mathrm{DEX}$ versus LEN + DEX estimates, with the new estimates for total incremental costs and quality-adjusted life-years being $-\$ 76,328$ and 1.19 , respectively. Thus, PAN $+\mathrm{BOR}+\mathrm{DEX}$ is still found to be dominant versus LEN + DEX in this comparison.

The second correction is to add the word "treatment" before the word "cost" in the "Discussion" section paragraph in which we discuss the Jakubowiak et al. study. ${ }^{2}$ The new sentence should read as follows:

"Finally, we note that 1 of the findings of the Jakubowiak et al. analysis appears to be counterintuitive, in that CFZ + LEN + DEX patients spend approximately 4 years in the postprogression state in the model versus approximately 3 years for LEN + DEX; however, the postprogression treatment costs for LEN+DEX are reported to be higher."

The overall postprogression costs are slightly higher for carfilzomib (CFZ) + LEN + DEX by $\$ 856$; however, the key point of the sentence remains valid, especially given the high cost of treating relapsed/refractory multiple myeloma; that is, patients receiving CFZ+LEN + DEX spent about 10 months more in the progression health state but had lower treatment costs and only slightly higher overall health state costs. This increase appears to be due to the use of different distributions of subsequent treatments by treatment arm and the assumption that subsequent treatments would stop after 17 cycles. Jakubowiak et al. suggest that they ran a scenario analysis using the same distribution, but the results of this scenario analysis are not provided.

We take this opportunity to point out an additional issue with the Jakubowiak et al. analysis. Their base case analysis uses 2 different health state utility values for the postprogression health state, even though patients in this health state should be relatively homogenous with respect to their healthrelated quality of life while in the same health state-that is, the patients will have already progressed on CFZ + LEN + DEX and LEN +DEX and would now be assumed to be treated similarly. Notably, the values applied to the CFZ + LEN + DEX intervention group after progression were higher than those for LEN + DEX (0.664 vs. 0.643). We note this additional bias in response to Ailawadhi et al.'s suggestion that "the cost-effectiveness of CFZ +DEX versus BOR +DEX has been unequivocally established," with reference to the Jakubowiak et al. study. ${ }^{2}$

Ailawadhi et al. also suggested additional limitations to the network meta-analysis (NMA) used in our study in terms of comparability. We stated in our article that "trial populations were similar with respect to age, ECOG performance status, ISS stage, receipt of previous stem cell transplant, and number and distribution of previous regimens." We acknowledged that definitions of disease risk varied. Other studies have used standard and comparable methods to assess PFS. Because PFS was unavailable for MM-009 and MM-010, time-to-progression was used; this approach has been used in other indirect comparisons of the agents of interest, including in the NICE panobinostat review cited by Ailawakhi et al. in their letter. Other recently published NMAs of relapsed/refractory multiple myeloma have taken a similar approach and have included a similar study set in their networks. ${ }^{3-6}$

In response to the comment that our results lack face validity because "LEN + DEX is estimated to be less effective than $\mathrm{BOR}+\mathrm{DEX}$ even though all listed trials show that LEN + DEX achieved double the PFS than BOR+DEX," we note that $\mathrm{LEN}+\mathrm{DEX}$ is not estimated to be less effective than BOR + DEX. The NMA did not show the 2 regimens to be statistically different. Importantly, trials of LEN +DEX have had follow-up durations that were 2-3 times longer than the BOR+DEX trials and were treat-to-progression, whereas BOR+DEX was given for a fixed number of cycles. Furthermore, and as we have already mentioned, we have corrected the PFS hazard ratio for $\mathrm{PAN}+\mathrm{BOR}+\mathrm{DEX}$ versus $\mathrm{LEN}+\mathrm{DEX}$, which now shows that the credible range includes 1 . We also note that our study has substantial cautionary language about this result, as shown in this example from the article: 